\title{
Quantitative analysis of antimicrobial use on British dairy farms
}

Robert M Hyde, BVM BVS AHFEA MRCVS ${ }^{1}$, John G Remnant, BVSc CertAVP DipECBHM FHEA MRCVS ${ }^{1}$, Andrew J Bradley, MA VetMB DCHP DipECBHM PhD MRCVS ${ }^{1,2}$, James E Breen, BVSc DCHP PhD MRCVS ${ }^{1,2}$, Christopher D Hudson, BVScPhD DCHP MRCVS ${ }^{1}$, Peers L Davies, MA VetMB PhD MRCVS ${ }^{1}$, Tom Clarke, BVSc MRCVS ${ }^{3}$, Yvonne Critchell ${ }^{3}$, Matthew Hylands, BVMBVS MRCVS4, Emily Linton, BVSc CertAVP(Cattle) MRCVS ${ }^{5}$, Erika Wood, BSc (Hons) ${ }^{6}$ Martin J Green, BVSc DCHP DipECBHM PhD MRCVS ${ }^{1}$

*Corresponding author; svxrh1@nottingham.ac.uk, University of Nottingham, School of Veterinary Medicine and Science, Sutton Bonington, Leicestershire LE12 5RD

${ }^{1}$ University of Nottingham, School of Veterinary Medicine and Science, Sutton Bonington, Leicestershire LE12 5L

${ }^{2}$ Quality Milk Management Services Ltd, Cedar Barn, Easton Hill, Easton, BA5 1DU

${ }^{3}$ Synergy Farm Health Ltd, West Hill Barns, Evershot, Dorchester, DT2 OLD

${ }^{4}$ Lambert Leonard and May (Lancs), 136 Whittingham Ln, Broughton, Preston, PR3 5DD

${ }^{5}$ Torch Farm Vets, 15 Habat Enterprise Park, Clovelly Road Industrial Estate, Bideford EX39 3HN

${ }^{6}$ Scarsdale Farm and Equine, Markeaton Lane, Derby, DE22 4NH 


\begin{abstract}
Antimicrobial resistance has been reported to represent a growing threat to both human and animal health, and concerns have been raised around levels of antimicrobial usage (AMU) within the livestock industry. To provide a benchmark for dairy cattle AMU and identify factors associated with high AMU, data from a convenience sample of 358 dairy farms were analysed using both mass-based and dose-based metrics following standard methodologies proposed by the European Surveillance of Veterinary Antimicrobial Consumption project. Metrics calculated were mass $(\mathrm{mg})$ of antimicrobial active ingredient per population correction unit (mg/PCU), defined daily doses (DDDvet) and defined course doses (DCDvet). AMU on dairy farms ranged from 0.36 to $97.79 \mathrm{mg} / \mathrm{PCU}$, with a median and mean of 15.97 and $20.62 \mathrm{mg} / \mathrm{PCU}$, respectively. Dose-based analysis ranged from 0.05 to 20.29 DDDvet, with a median and mean of 4.03 and 4.60 DDDvet, respectively. Multivariable analysis highlighted that usage of antibiotics via oral and footbath routes increased the odds of a farm being in the top quartile (>27.9 mg/PCU) of antimicrobial users. While dairy cattle farm AMU appeared to be lower than UK livestock average, there were a selection of outlying farms with extremely high AMU, with the top 25 per cent of farms contributing greater than 50 per cent of AMU by mass. Identification of these high use farms may enable targeted AMU reduction strategies and facilitate a significant reduction in overall dairy cattle AMU.
\end{abstract}

http://dx.doi.org/10.1136/vr.104614 


\section{Introduction}

Antimicrobial resistance (AMR) has been reported to represent a growing threat to both human and animal health,,-1 with the inappropriate use of antimicrobials in human and animal fields being considered responsible for the emergence of resistance in bacteria. ? Concerns have been raised regarding the zoonotic transfer of resistant bacteria from livestock to human beings,,$\underline{3}$ with potential routes of transfer including food products, $\underline{4}$ direct contact $\underline{5}$ and via environmental contamination. $\underline{6}$ There are however many knowledge gaps in livestock-human AMR transfer routes,,-7 and links between on-farm antimicrobial usage (AMU) and the increased transfer of major foodborne drug-resistant organisms such as Campylobacter spp and Salmonella spp have not been found. $\underline{89}$

Remarkably strong interactions between AMU and AMR in livestock have been described,, 0 and UK Government-commissioned reports have called for a reduction in antimicrobial use within the livestock industry. .11 There are numerous metrics available to measure veterinary AMU, which have great impact on both the AMU figures obtained and consequent influence on AMU reduction policy,,$\underline{12}$ and international recommendations have been provided for the most suitable metrics to monitor both human and veterinary AMU. 13 The UK Government has called for an AMU reduction target from $62 \mathrm{mg}$ per population correction unit (mg/PCU) in 2013 to less than $50 \mathrm{mg} / \mathrm{PCU}$ as an average across all livestock sectors by $2018, \underline{14}$ and total livestock AMU figures for the UK indicate this target has already been reached. .5 Farm-level AMU monitoring studies in dairy cattle have been conducted in several countries including the USA, $\stackrel{16}{ }$ Canada,,$\underline{17}$ New Zealand $\frac{18}{}$ and the Netherlands $\frac{19}{}$; however, to date, no detailed farm-level AMU studies identifying factors associated with high AMU have been conducted in the UK dairy industry.

Therefore, this study aims to provide an evaluation of the levels of AMU in a convenience sample of British dairy farms over a 12-month period and to identify factors associated with high usage on these farms.

\section{Methods}

\section{Sources of data}

Data were collected from both veterinary practice records and electronic onfarm records between January 1, 2016 and December 31, 2016. A convenience sample of four farm animal veterinary practices in the south, midlands and north of England was identified after an expression of interest in AMU monitoring, and anonymised antimicrobial sales data were provided for all dairy farm clients between January 1, 2016 and December 31, 2016. Cow numbers were estimated for each farm using electronic cattle movement records (using the British Cattle Movement Service Cattle Tracing System, with animals being $>24$ months and female) for two practices or otherwise directly from farmer estimates of average adult dairy cow numbers. Farms 
with any other livestock on farm (ie, sheep or beef enterprises) were excluded from the study to ensure antimicrobials purchased were solely for dairy cattle.

AMU records were obtained from a separate group of farms recording medicines usage electronically on farm, which were enrolled in regular milk recording with a laboratory based in the south of England (Quality Milk Management Services). Farmer-recorded electronic medicines records, cow numbers and veterinary practice ID were extracted from on-farm software.

Antimicrobial sales records were collected for 332 farms, with 40 farms being excluded due to the presence of a livestock enterprise other than dairy, resulting in a final data set of 292 farms. Electronically recorded AMU records were collected from 70 farms, with 4 farms being excluded due to data recording errors, resulting in a final data set of 66 farms. These two data sets were combined to provide a total of 358 farms, with antimicrobial data for 81,640 dairy cows (Table 1), representing $~ 3.6$ per cent of total dairy farms and $\sim 7.0$ per cent of total dairy cows in England. 20 Farm size ranged from 21 to 2080 , with a mean herd size of 228 . The four practices supplying antimicrobial sales data were dominated by one practice providing information for 182 farms, with the other three practices supplying data for the remaining 110 farms (Table 1$)$.

Practice 1 Practice 2 Practice 3 Practice 4 All Sales All Use All farms

\begin{tabular}{r|rrrrrrr} 
Min & 70 & 37 & 21 & 25 & 21 & 50 & 21 \\
Max & 340 & 550 & 562 & 2080 & 2080 & 1290 & 2080 \\
Median & 160 & 120 & 199 & 179 & 171 & 183 & 172 \\
Mean & 178 & 160 & 199 & 252 & 223 & 249 & 228 \\
Total dairy cows & 3551 & 8807 & 6973 & 45892 & 65223 & 16417 & 81640 \\
Total dairy farms & 20 & 55 & 35 & 182 & 292 & 66 & 358
\end{tabular}

TABLE 1: Cow numbers per farm for four veterinary practices that provided antimicrobial sales data ('All Sales') and from a group of farms recording medicines usage electronically ('All Use')

\section{Calculation of AMU}

Total mass $(\mathrm{mg})$ of antimicrobial active ingredient per population correction unit (mg/PCU) was calculated using the European Surveillance of Veterinary Antimicrobial Consumption (ESVAC) standard methodology,, 1 using a standardised adult dairy cow bodyweight of $425 \mathrm{~kg}$. Youngstock and slaughtered animals were not included in the calculation of PCU, and topical sprays were not included in the calculation of antibiotic mass.

To facilitate comparison between different monitoring metrics, three separate metrics were calculated for both sales and usage data: $\mathrm{mg} / \mathrm{PCU}$, defined daily doses (DDDvet) and defined course doses (DCDvet). Critically important 
antibiotics (CIAs) were defined as third-generation or fourth-generation cephalosporins and fluoroquinolones. $\underline{22}$

DDDvet calculations followed standard ESVAC/European Medicines Agency (EMA) methodology 21 to provide an estimation of AMU while taking into account differences in dosing between antimicrobial products. Oral and parenteral antibiotic formulations were calculated per kilogram of animal (adult dairy cow weight being $425 \mathrm{~kg}$ ), and intrauterine and lactating cow intramammary doses (IMM) were calculated per cow. Antibiotic dry cow therapy (DCT) was excluded from DDDvet calculations as per ESVAC/EMA methodology, as were topical antibiotic therapies (including antibiotic footbathing).

DCDvet calculations were performed similarly, calculating oral and parenteral doses per kilogram, intrauterine and lactating cow intramammary courses per cow, also including dry cow intramammary therapy, with four tubes per cow being equal to a single course.

Basic descriptive statistics (range, median, mean) were performed for all metrics calculated, and total mass of antimicrobials by route was also calculated and illustrated graphically. Calculations were repeated for CIAs. Total AMU/sales per farm were graphically illustrated for each practice to identify any patterns within practices. This was also repeated for CIAs. Basic descriptive statistics and graphical illustrations were conducted using Microsoft Excel 2016 and R V.3.4.0 statistical software. $\underline{23}$

Statistical analysis

To investigate factors that influenced farms being high antimicrobial users, multilevel logistic regression models were developed using MLwiN, $\underline{24}$ with farms in the top 25 per cent of antimicrobial users

( $>27.9 \mathrm{mg} / \mathrm{PCU},>5.88$ DDDvet or $>2.32 \mathrm{DCD} v e t)$ as a binary outcome variable (highest 25 per cent or not). Categorical variables were created for herd size (small $<136$, medium 136-236, large $>236$ ), and a binary variable of route usage was created illustrating either 'used' or 'did not use' for each route category (parenteral, IMM, DCT, footbath, intrauterine, oral, pessary). Models were constructed using a backwards stepwise approach until variables that remained in the model were significant at $P<0.05$, and model fit was checked using a modified Hosmer-Lemeshow method $\stackrel{25}{ }$. A multilevel structure was employed to allow for correlations between farms within veterinary practices, with veterinary practice being included as a random effect. Parameters were estimated using iterative generalised least squares. Final parameter estimates were generated using Markov Chain Monte Carlo (MCMC), with Gibbs sampling to avoid potential parameter bias. $\underline{26} \mathrm{~A}$ burn in chain of 500 iterations with a monitoring chain of 10,000 iterations was used, and $\mathrm{MCMC}$ chains were visually checked to ensure convergence. To estimate the proportional reduction in high AMU farms that would occur if significant risk factors were removed, population attributable fractions (PAFs) were calculated for significant model covariates. $\underline{27}$ PAFs and 95 per cent confidence 
intervals were calculated directly from MCMC chains using $\mathrm{R}$ statistical software (R V.3.4.0 2017).

\section{Results}

Antimicrobial sales to dairy farms ranged from 0.36 to $97.79 \mathrm{mg} / \mathrm{PCU}$, with a median and mean of 17.25 and $22.11 \mathrm{mg} / \mathrm{PCU}$, respectively. DDDvet for sales data ranged from 0.05 to 20.29 doses, with a median and mean of 4.22 and 4.84 , respectively. DCDvet for sales data ranged from 0.01 to 6.74 courses, with a median and mean of 1.73 and 1.93, respectively.

Farmer-recorded AMU ranged from 1.20 to $48.48 \mathrm{mg} / \mathrm{PCU}$, with a median and mean of 11.42 and $14.06 \mathrm{mg} / \mathrm{PCU}$, respectively. DDDvet for usage data ranged from 0.14 to 16.09 doses, with a median and mean of 3.24 and 3.56, respectively, and DCDvet for usage data ranged from 0.33 to 5.33 courses, with a median and mean of 1.55 and 1.58, respectively.

Of all the farms included in this study, the median AMU was $15.97 \mathrm{mg} / \mathrm{PCU}$, or 4.03 DDDvet and 1.69 DCDvet. The mean AMU from all the farms in the study was $20.62 \mathrm{mg} / \mathrm{PCU}$, or 4.60 DDDvet and 1.86 DCDvet (Table 2). 
Source (Farm numbers)

\begin{tabular}{|c|c|c|c|c|c|c|c|c|}
\hline & & $\begin{array}{l}\text { Practice } \\
1(20)\end{array}$ & $\begin{array}{l}\text { Practice } \\
2 \text { (55) }\end{array}$ & $\begin{array}{l}\text { Practice } \\
\mathbf{3} \text { (35) }\end{array}$ & $\begin{array}{l}\text { Practice } \\
4 \text { (182) }\end{array}$ & $\begin{array}{l}\text { All } \\
\text { Sales } \\
(292)\end{array}$ & $\begin{array}{l}\text { All } \\
\text { Use } \\
(66)\end{array}$ & $\begin{array}{l}\text { All } \\
\text { farms } \\
(358)\end{array}$ \\
\hline \multirow[t]{4}{*}{$m g / P C U$} & Min & 7.84 & 0.36 & 5.02 & 0.55 & 0.36 & 1.20 & 0.36 \\
\hline & Max & 85.92 & 97.79 & 96.47 & 70.76 & 97.79 & 48.48 & 97.79 \\
\hline & Median & 19.86 & 18.62 & 32.41 & 15.55 & 17.25 & 11.42 & 15.97 \\
\hline & Mean & 26.85 & 22.59 & 38.07 & 18.37 & 22.11 & 14.06 & 20.62 \\
\hline \multirow[t]{4}{*}{ DDDvet } & Min & 0.90 & 0.07 & 0.97 & 0.05 & 0.05 & 0.14 & 0.05 \\
\hline & Max & 17.23 & 18.59 & 12.78 & 20.29 & 20.29 & 16.09 & 20.29 \\
\hline & Median & 6.39 & 5.01 & 4.89 & 3.71 & 4.22 & 3.24 & 4.03 \\
\hline & Mean & 6.48 & 6.04 & 5.57 & 4.15 & 4.84 & 3.56 & 4.60 \\
\hline \multirow[t]{4}{*}{ DCDvet } & Min & 0.26 & 0.02 & 0.31 & 0.01 & 0.01 & 0.33 & 0.01 \\
\hline & Max & 6.62 & 6.74 & 4.45 & 6.63 & 6.74 & 5.33 & 6.74 \\
\hline & Median & 2.35 & 2.23 & 1.81 & 1.57 & 1.73 & 1.55 & 1.69 \\
\hline & Mean & 2.61 & 2.45 & 2.09 & 1.67 & 1.93 & 1.58 & 1.86 \\
\hline
\end{tabular}

TABLE 2:

Antimicrobial usage ( $\mathrm{mg} / \mathrm{PCU}, D D D v e t, D C D v e t)$ for four veterinary practices that provided antimicrobial sales data ('All Sales') and from a group of farms recording medicines usage electronically ('All Use')

Antibiotic sales and usage largely consisted of injectable treatments, comprising 78.1 per cent of total antibiotic mass used or sold (Fig 1$)$. Lactating cow intramammary treatments represented 6.5 per cent of antimicrobial active ingredient mass $(\mathrm{mg})$, with DCT representing 6.0 per cent. Off-licence antibiotic footbath powders represented 4.5 per cent of total $\mathrm{mg}$, and oral powders, intrauterine treatments and pessaries combined represented 5.0 per cent. Beta lactams made up the bulk of antimicrobials, totalling 42.8 per cent of $\mathrm{mg}$, with aminoglycosides totalling 20.9 per cent. CIAs totalled 3.3 per cent of $\mathrm{mg}$, with third-generation or fourth-generation cephalosporins and fluoroquinolones comprising 2.5 per cent and 0.8 per cent of $\mathrm{mg}$, respectively, and representing a mean farm AMU of 4.7 per cent of mg/PCU ranging from 0 to 47.6 per cent. Due to the relatively lower dosing requirements of CIAs, CIAs represented a higher proportion of doses administered, ranging from 0 to 90.1 per cent DDDvet and from 0 to 89.0 per 
cent DCDvet, representing a mean of 17.8 per cent DDDvet and 14.9 per cent DCDvet, respectively.

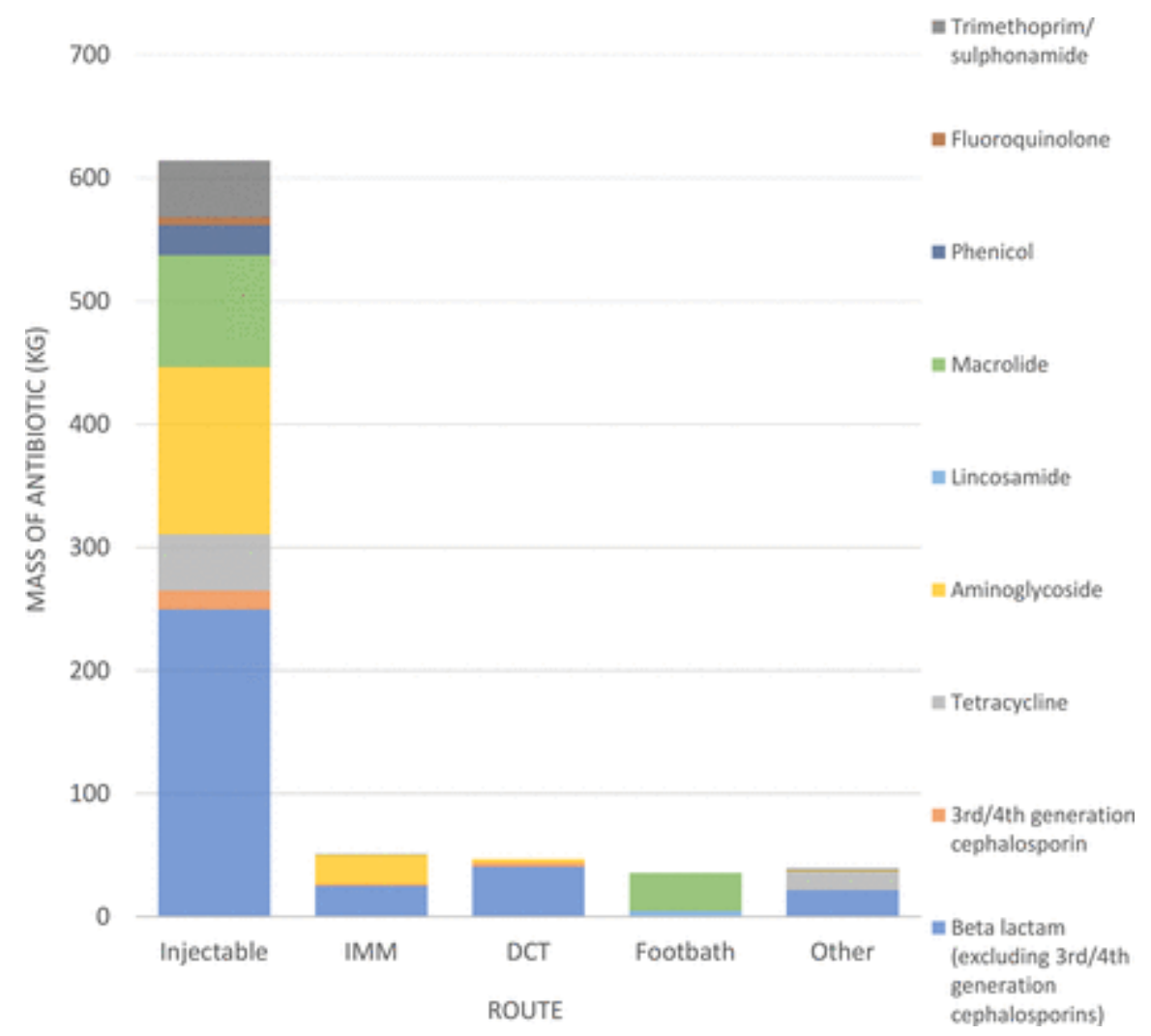

FIG 1: Total mass $(\mathrm{kg})$ of antimicrobial classes from sales data to farms $(n=292)$ from four veterinary practices and for usage data from farms $(n=66)$ recording medicines usage electronically on farm. DCT, dry cow therapy; IMM, intramammary lactating cow therapy.

The majority of farms used/purchased antibiotic IMM and DCT products (345 (96 per cent) and 338 (94 per cent), respectively), and all farms used/purchased injectable antibiotic products. Farms also used/purchased the following products: intrauterine antibiotics (184 (51 per cent)), antibiotic pessaries (117 (33 per cent)), antibiotic footbaths (98 (27 per cent)) and oral antibiotics (18 (5 per cent)). Multivariable regression modelling illustrated that the use of oral and footbath antibiotics significantly increased the odds of a farm being in the top 25 per cent of antimicrobial users, measured by mg/PCU (Table 3). Other variables such as herd size and usage of other routes of antimicrobial administration (parenteral, IMM, DCT, intrauterine and pessary) did not remain in the model. 


\begin{tabular}{|c|c|c|c|c|c|c|}
\hline Outcome variable & $\begin{array}{l}\text { Explanatory variable } \\
\text { (N) }\end{array}$ & $\begin{array}{l}\text { Odds } \\
\text { ratio }\end{array}$ & $95 \% \mathrm{Cl}$ & $\begin{array}{l}\text { P- } \\
\text { value }\end{array}$ & $\begin{array}{l}\text { Population } \\
\text { attributable } \\
\text { fraction (\%) }\end{array}$ & $95 \% \mathrm{Cl}$ \\
\hline \multirow[t]{2}{*}{$\begin{array}{l}\text { Top 25\% AMU } \\
\text { (mg/PCU) (n=90) }\end{array}$} & $\begin{array}{l}\text { Oral antibiotics used } \\
(n=13)\end{array}$ & 9.43 & $(2.76-32.27)$ & $<0.001$ & 8.4 & $(3.03-14.00)$ \\
\hline & $\begin{array}{l}\text { Antibiotic footbath } \\
\text { used }(n=39)\end{array}$ & 2.75 & $(1.46-5.17)$ & 0.001 & 20.28 & (8.99-33.02) \\
\hline
\end{tabular}

TABLE 3: Logistic regression model of factors affecting the odds of farms $(n=358)$ being in the highest 25 per cent of antimicrobial users (as measured by $\mathrm{mg} / \mathrm{PCU}$ )

\section{Discussion}

Given that cattle represents 25 per cent of total livestock mass in the UK as

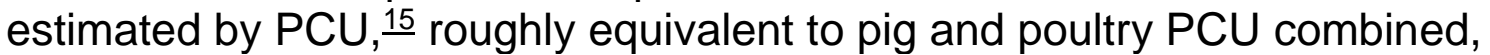
AMU within the cattle industry plays an important role in the UK's overall AMU. The results from this study indicate that AMU in dairy cattle is likely to be significantly lower than overall UK livestock AMU of $45 \mathrm{mg} / \mathrm{PCU}$ in

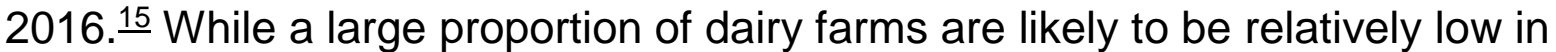
terms of $A M U$, in this data set there exists an outlying population of farms that exhibit a relatively high usage of antimicrobials, with the highest 25 per cent of antimicrobial users representing 52 per cent of the total mass $(\mathrm{mg})$ of antimicrobials. Establishing monitoring methods and targets will be essential to identify high antimicrobial users, and an understanding of factors that influence relatively high AMU will allow targeted AMU reduction interventions.

Injectable preparations represented the majority of antimicrobial use/sales mass, with the remainder being predominantly IMM, DCT and footbath routes (Fig 1). Of injectable products 3.5 per cent by mass were CIAs, and injectable treatments largely comprised beta lactams ( 40.6 per cent) and aminoglycosides (22.1 per cent) by mass, representing the common usage of both penicillin-based antibiotics and penicillin streptomycin combinations. Differences between monitoring metrics resulted in important differences between proportions of AMU attributed to various routes of therapeutic application, which has the potential to influence national-level and farm-level protocols to reduce AMU. Mass-based AMU monitoring (ie, mg/PCU) appears heavily influenced by parenteral therapy, potentially incentivising the reduction of parenteral therapy as a priority with IMM treatments playing a small role in overall mg/PCU as previously reported. .2 Dose-based metrics (ie, DDDvet, DCDvet), however, highlight IMM therapies as major contributors to total AMU and may encourage reductions in IMM antimicrobials as a priority. While there is a degree of correlation between the monitoring methodologies, there are high AMU farms that would not be detected if a single monitoring metric was used (Fig 2), with high IMM and high footbath AMU being particularly poorly 
represented when using mass-based or dose-based methodology, respectively. Given that monitoring methodologies will potentially incentivise reductions in specific treatment routes, it may be prudent to aim for AMU reductions as monitored by both mass-based and dose-based metrics.

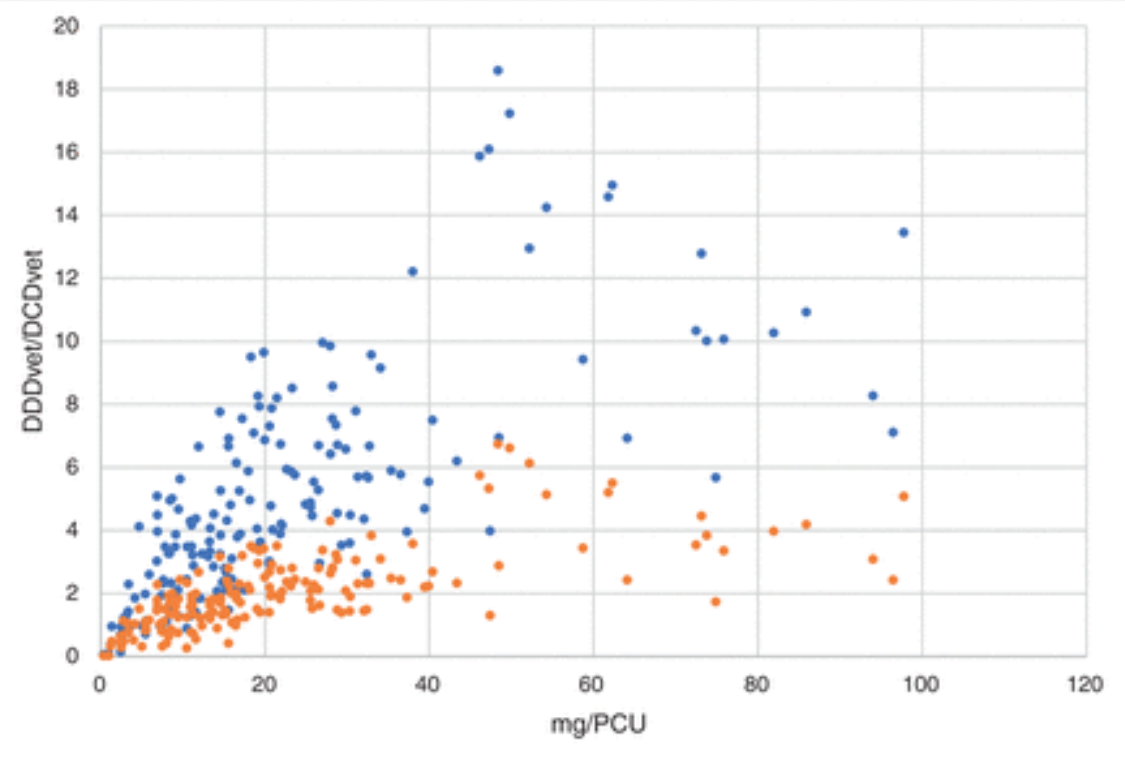

FIG 2: Antimicrobial usage measured by mg/PCU compared with DDDvet (blue) and DCDvet (orange) from sales data to farms from four veterinary practices and for usage data from farms recording medicines usage electronically. The total number of farms is 358. DCDvet, defined course doses; DDDvet, defined daily doses; PCU, population correction unit.

The reduction/cessation of usage of antibiotics critically important for human health has received great attention within the livestock industry. $\frac{11}{1}$ The current study demonstrated that CIAs represent a mean of 4.7 per cent mg/PCU used on dairy farms, which is high compared with other species (1 per cent of all livestock mg/PCU in 2016탐. When employing dose-based methodology, the mean farm CIA usage represented 17.8 per cent of DDDvet and 14.9 per cent of DCDvet, respectively, a difference largely due to generally lower dosing requirements of CIAs. Since pressure on the livestock industry to reduce CIA usage will continue to grow, the usage of CIAs on dairy farms will come under scrutiny. To reduce AMU across the livestock industry, it will be important to provide industry-specific targets both in terms of reducing overall usage and specific therapeutics such as CIAs.

Monitoring dairy farm AMU to identify high antimicrobial users would enable targeted interventions to facilitate the most effective reductions in overall AMU. The use of oral and footbath antibiotics on farms appears to increase the odds of farms being in the highest 25 per cent of antimicrobial users (mg/PCU), possibly indicating the effects of group medication protocols on increased AMU. This study also illustrated that while most dairy cattle farms do not employ these methods of medication, those that do can dramatically increase overall mass-based AMU (Fig 3). High levels of injectable antibiotic usage are also likely to be a significant driver of overall AMU as measured by 
$\mathrm{mg} / \mathrm{PCU}$, but due to all farms using injectable products, injectable usage was not retained in the final multivariable model. Targeting the reduction of AMU among the minority of high antimicrobial users while maintaining high standards of health, welfare and production may be a fast and effective first step to reduce AMU at farm, practice and national levels.

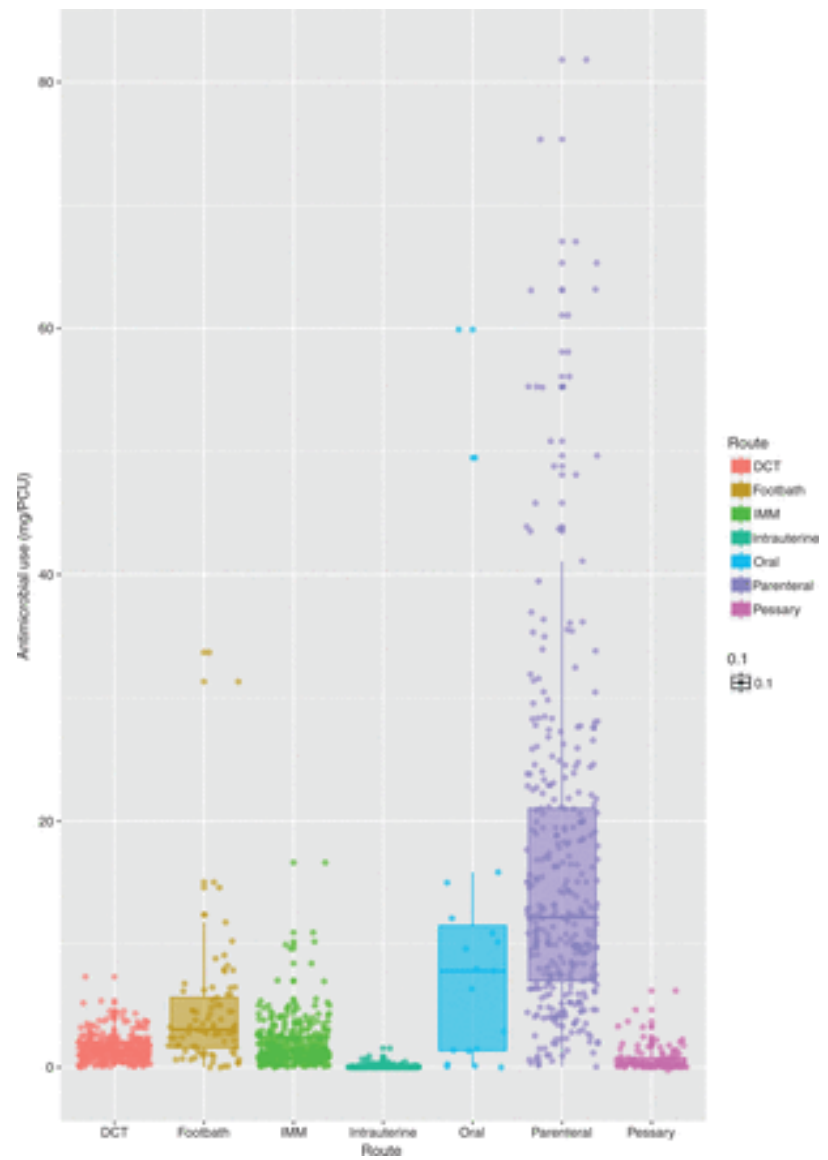

FIG 3: Antimicrobial usage ( $\mathrm{mg} / \mathrm{PCU}$ ) by route from sales data to farms from four veterinary practices and for usage data from farms recording medicines usage electronically. Points represent antimicrobial usage (mg/PCU) for each farm by route, with box plots illustrating interquartile range and median. The total number of farms is 358. DCT, dry cow therapy; $I M M$, intramammary lactating cow; PCU, population correction unit.

UK legislation requires medicines administered to livestock to be recorded on farm and the sales of all veterinary products to livestock businesses to be recorded by veterinary practices, $\underline{28}$ resulting in two readily available data sources for farm-level AMU monitoring. Farmer-recorded usage will potentially under-report actual usage on farm, as farmer-inputted medicines use has previously been shown to be an under-representation when compared with medicines sales. $\frac{1929}{29}$ This may in part be related to medicines being bought in bulk and not all being used (and therefore not recorded), alongside errors in updating medicines records. In contrast, practice sales records capture all antimicrobials sold to a farm, and therefore may over-report AMU due to antimicrobials not ultimately used. Since important differences in recording methodology and terminology exist between both farms and practices, standardisation of recording protocol will be essential in national monitoring 
efforts, and caution should be taken when comparing farmer-recorded usage with antimicrobial sales figures.

AMU comparisons between monitoring studies can be challenging, with many factors influencing both the numerator (mg or doses) and the denominator (PCU) populations, depending on methodology used. The standard weight of an adult cow will have a significant bearing on denominator population, with studies using 'weight at time of treatment' such as $425 \mathrm{~kg} \underline{21}$ potentially underestimating weight when compared with live cattle weights such as $600 \mathrm{~kg} \frac{1719}{10}$ and $680 \mathrm{~kg}, \frac{16}{16}$ resulting in AMU overestimation. The inclusion/exclusion of youngstock also has bearing on the denominator population, and the inclusion of youngstock AMU in this study despite exclusion of youngstock PCU may slightly overestimate AMU as previously described, $\frac{1719}{19}$ although $A M U$ for calves have previously been reported to likely represent a small proportion of total AMU on dairy farms. $\frac{19}{}$ DCT recording, however, can have a significant bearing on dose-based methodologies, and while DCT is excluded from DDDvet,, 1 doses both per cow and per teat have been previously reported. $\frac{1719}{19}$ Antibiotic footbathing has a dramatic effect on $\mathrm{mg} / \mathrm{PCU}$, however is not accounted for when applying DDDvet or DCDvet methodologies. It is challenging to provide clear guidance as to the 'dosage' applied to each animal, and anecdotally $\sim 100 \mathrm{~g}$ of antibiotic powder might be advised to treat around 100 cows via footbath. Using a dosage of $1 \mathrm{~g}$ of product per cow as a daily dose allows an estimation into the effect of footbathing on the dose-based metrics, which would increase mean farm AMU by over 10 per cent from 4.6 to 5.1 DDDvet.

ESVAC/EMA calculations based on live dairy cattle and slaughter weights have been largely designed to facilitate national/international monitoring, and are not intended for farm-level comparisons. Since the number of youngstock on dairy farms varies widely, it can be challenging to directly compare farms due to the variation in PCU not captured using ESVAC/EMA methodology. Cattle births, deaths and movements in the UK are reported to the British Cattle Movement Service. $\underline{.0}$ Using this database, it should be possible to identify a relatively accurate estimation of PCU present on each holding by assigning weights to each age category, calculating number of days spent in each weight class and providing an overall estimation as to $\mathrm{kg} / \mathrm{year}$ at risk. This methodology should reduce PCU estimation errors between dairy farms with variations in youngstock rearing and would also be applicable to the beef industry, where a wide range of farm management systems make farm-level AMU comparisons challenging.

The mean herd size (228) in this study was larger than average for dairy herds in England (145릉), although herd size did not appear to be associated with AMU in this study. The study population is based on a convenience sample and cannot be assumed to be a sample entirely representative of British dairy farms. There is potential for selection bias, with veterinary practices contributing data having previously expressed their interest and proactive approach to AMU monitoring, and farms identified via milk recording records 
already being engaged in electronic medicines recording. Despite this, there appears to be clear similarities between practices, with most farms in each practice representing a relatively low usage of antimicrobials, with a minority of farms comprising a major proportion of total AMU (Figs 4 and 5 ).

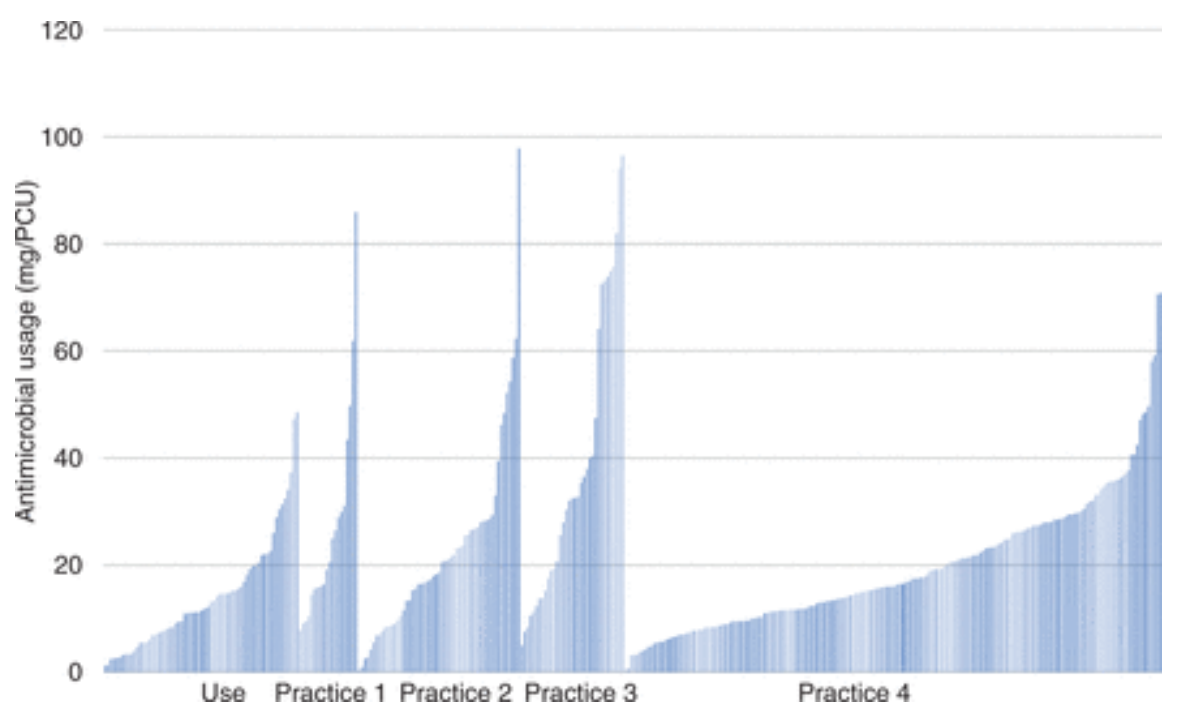

FIG 4: Antimicrobial usage ( $m g / P C U)$ from sales data to farms $(n=292)$ from four veterinary practices and for usage data from farms $(n=66)$ recording medicines usage electronically on farm. PCU, population correction unit.

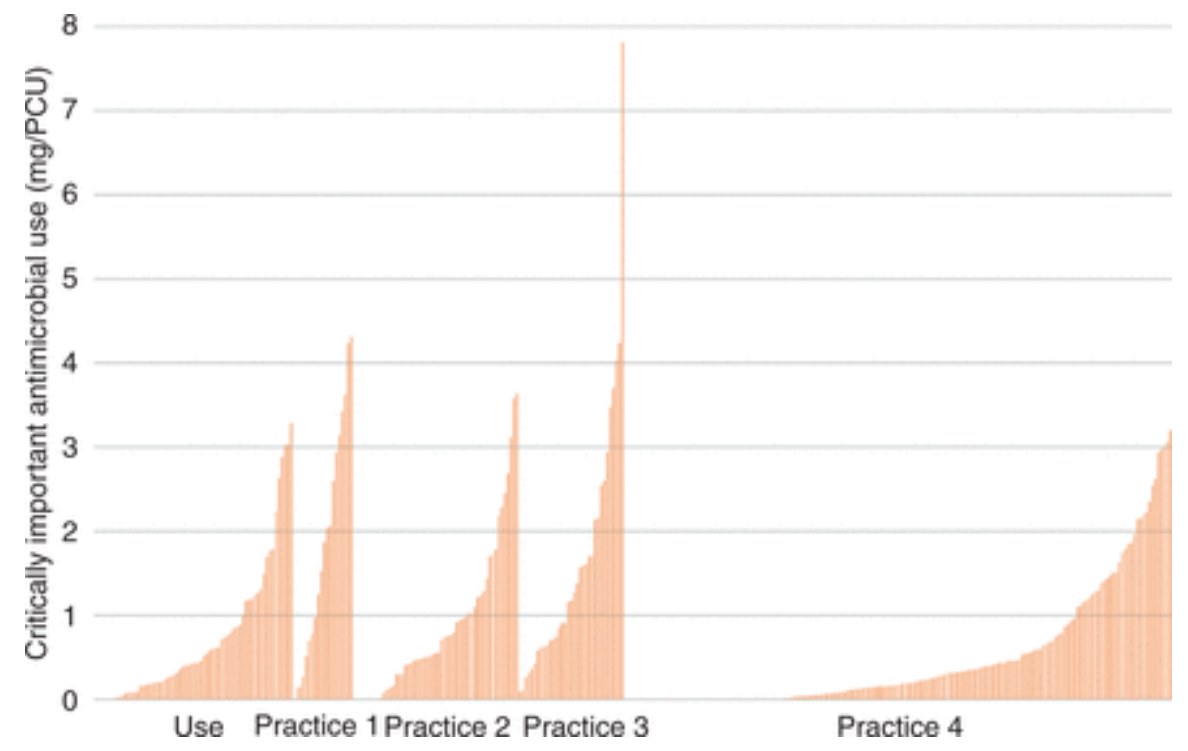

FIG 5: Critically important antimicrobial usage ( $\mathrm{mg} / \mathrm{PCU}$ ) from sales data to farms $(n=292)$ from four veterinary practices and for usage data from farms $(n=66)$ recording medicines usage electronically on farm. PCU, population correction unit.

Pressure will no doubt continue to mount on the livestock industry to monitor and reduce AMU, and it will be important to monitor AMU at farm level across all livestock sectors, employing both mass-based and dose-based metrics. This study provides a benchmark for dairy cattle AMU in Britain and identifies several factors associated with high AMU. While this study suggests that most dairy farms exhibit lower AMU than the UK livestock average, there remains an outlying population of farms with high levels of AMU. Identifying high AMU 
farms at a practice or national level will enable AMU reduction efforts to be efficiently targeted, and future studies should include the effect of such interventions on levels of both AMU and AMR. A proactive veterinary approach to herd health management to reduce disease incidence will result in fewer antimicrobial treatments being required and will play an important role in the reduction of dairy cattle AMU.

\section{Acknowledgments}

The authors would like to thank all veterinary practices involved, Quality Milk Management Services and all dairy farmers participating in the study. 
References

1 Wernli D, Haustein T, Conly J, et al. A call for action: the application of The International Health Regulations to the global threat of antimicrobial resistance. PLoS Med 2011;8:e1001022.

2 Levy SB, Marshall B. Antibacterial resistance worldwide: causes, challenges and responses. Nat Med 2004;10:S122-S129.

3 Marshall BM, Levy SB. Food animals and antimicrobials: impacts on human health. Clin Microbiol Rev 2011;24:718-33.

4 Lazarus B, Pater son DL, Mollinger JL, et al. Do human extraintestinal Escherichia coli infections resistant to expanded-spectrum cephalosporins originate from food-producing animals? A systematic review. Clin Infect Dis 2015;60:439-52.

5 Klous G, Huss A, Heederik DJJ, et al. Human-livestock contacts and their relationship to transmission of zoonotic pathogens, a systematic review of literature. One Health 2016;2:65-76.

6 Berendonk TU, Manaia CM, Merlin C, et al. Tackling antibiotic resistance: the environmental framework. Nat Rev Microbiol 2015;13:310-7.

7 Horigan V, Kosmider RD, Horton RA, et al. An assessment of evidence data gaps in the investigation of possible transmission routes of extended spectrum $\beta$-lactamase producing Escherichia coli from livestock to humans in the UK. Prev Vet Med 2016;124:1-8.

8 Helke KL, McCrack in MA, Galloway AM, et al. Effects of antimicrobial use in agricultural animals on drug-resistant foodborne salmonellosis in humans: A systematic literature review. Crit Rev Food Sci Nutr 2017;57:472-88.

9 McCrack in MA, Helke KL, Galloway AM, et al. Effect of antimicrobial use in agricultural animals on drug-resistant foodborne campylobacteriosis in humans: a systematic literature review. Crit Rev Food Sci Nutr 2016;56:2115-32.

10 Chantziaras I, Boyen F, Callens B, et al. Correlation between veterinary antimicrobial use and antimicrobial resistance in food-producing animals: a report on seven countries. J Antimicrob Chemother 2014;69:827-34.

$11 \mathrm{O}^{\prime} \mathrm{Ne}$ ill J. Antimicrobials in agriculture and the environment: reducing unnecessary use and waste the review on antimicrobial resistance, 2015.

12 Hyde R, Green M, Remnant J, et al. Tool to measure antimicrobial use on farms. Vet Rec 2017;180:183.1-183.

13 Collineau L, Belloc C, St ärk KD, et al. Guidance on the selection of appropriate indicators for quantification of antimicrobial usage in humans and animals. Zoonoses Public Health 2017;64:16584.

14 Department of Health. Government response to the review on antimicrobial resistance, 2016.

15. VMD. Veterinary Antimicrobial Resistance and Sales Surveillance 2016: Veterinary Antimicrobial Resistance and Sales Surveillance, 2017.

16 Pol M, Ruegg PL. Treatment practices and quantification of antimicrobial drug usage in conventional and organic dairy farms in Wisconsin. J Dairy Sci 2007;90:249-61. 
17 Saini V, McClure JT, Léger D, et al. Antimicrobial use on Canadian dairy farms. J Dairy Sci 2012;95:1209-21.

18 Bryan M, Hea SY. A survey of antimicrobial use in dairy cows from farms in four regions of New Zealand. N Z Vet J 2017;65:93-8.

19 Kuipers A, Koops WJ, Wemmenhove H. Antibiotic use in dairy herds in the Netherlands from 2005 to 2012. J Dairy Sci 2016;99:1632-48.

20 AHDB. Dairy statistics: an insider's guide, 2016.

21 EMA. Principles on assignment of defined daily dose for animals (DDDA) and defined course dose for animals (DCDA), 2015.

22 EMA. Answers to the request for scientific advice on the impact on public health and animal health of the use of antibiotics in animals, 2014.

23 R core team. R: a language and environment for statistical computing, 2017.

24 Charlto n C. MLwiN version 3.00, 2017.

25 Hosmer DW, Lemeshow S, Sturd ivant RX. Applied logistic regression, 2013.

26 Browne WJ. MCMC estimation in MLwiN v3.00, 2017.

27 Rothman KJ, Greenland S. Modern epidemiology, Lippincott-Raven, 1998.

28 The veterinary medicines regulations, 2013.

29 González SM, Ste iner A, Gassner B, et al. Antimicrobial use in Swiss dairy farms: quantification and evaluation of data quality. Prev Vet Med 2010;95:50-63.

30 UK Government. The cattle identification regulations, 2007. 